\title{
Exact bosonization in arbitrary dimensions
}

\author{
Yu-An Chen 10 \\ California Institute of Technology, Pasadena, California 91125, USA
}

(Received 17 June 2020; revised 10 September 2020; accepted 14 September 2020; published 30 September 2020)

\begin{abstract}
We extend the previous results of exact bosonization, mapping from fermionic operators to Pauli matrices, in $2 \mathrm{D}$ and $3 \mathrm{D}$ to arbitrary dimensions. This bosonization map gives a duality between any fermionic system in arbitrary $n$ spatial dimensions and a class of $(n-1)$-form $\mathbb{Z}_{2}$ gauge theories in $n$ dimensions with a modified Gauss's law. This map preserves locality and has an explicit dependence on the second Stiefel-Whitney class and a choice of spin structure on the spatial manifold. A formula for Stiefel-Whitney homology classes on lattices is derived. In the Euclidean path integral, this exact bosonization map is equivalent to introducing a topological Steenrod square term to the space-time action.
\end{abstract}

DOI: 10.1103/PhysRevResearch.2.033527

\section{INTRODUCTION AND SUMMARY}

It is well known that every fermionic lattice system in 1D is dual to a lattice system of spins with a $\mathbb{Z}_{2}$ global symmetry (and vice versa). The duality is kinematic (independent of a particular Hamiltonian) and arises from the Jordan-Wigner transformation. Recently, it has been shown that any fermionic lattice system in $2 \mathrm{D}$ is dual to a $\mathbb{Z}_{2}$ gauge theory with an unusual Gauss's law [1]. The fermion can be identified with the flux excitation of the gauge theory, which is described by the Chern-Simons-like term $i \pi \int A \cup \delta A$ in the space-time action. The $2 \mathrm{D}$ duality is also kinematic. This approach has been generalized to 3D [2]. Every fermionic lattice system in $3 \mathrm{D}$ is dual to a $\mathbb{Z}_{2} 2$-form gauge theory with an unusual Gauss's law. Here, 2-form gauge theory means that the $\mathbb{Z}_{2}$ variables live on faces (2-simplices), while the parameters of the gauge symmetry live on edges (1-simplices). 2 -form gauge theories in $3+1 \mathrm{D}$ have local flux excitations, and the unusual Gauss's law ensures that these excitations are fermions. This Gauss's law can be described by the Steenrod square topological action $i \pi \int B \cup B+B \cup_{1} \delta B$. The form of the modified Gauss's law was first observed in Ref. [3]: A bosonization of fermionic systems in $n$ dimensions must have a global $(n-1)$-form $\mathbb{Z}_{2}$ symmetry with a particular 't Hooft anomaly. The standard Gauss's law leads to a trivial 't Hooft anomaly, so bosonization requires us to modify it in a particular way.

In this paper, we extend these results to arbitrary $n$ dimensions. We show that every fermionic lattice system in $n$ dimension is dual to a $\mathbb{Z}_{2}(n-1)$-form gauge theory with a modified Gauss's law. Our bosonization map is kinematic and local in the same sense as the Jordan-Wigner map: ${ }^{1}$ Every

\footnotetext{
${ }^{1}$ We only consider the locality preserving map here. Although Jordan-Wigner transformation can map a single fermionic operator into spins, it contains a string operator, which is highly nonlocal. Our bosonization map and Jordan-Wigner transformation both preserve the locality of observables in fermionic systems.
}

Published by the American Physical Society under the terms of the Creative Commons Attribution 4.0 International license. Further local observable on the fermionic side, including the Hamiltonian density, is mapped to a local gauge-invariant observable on the $\mathbb{Z}_{2}$ gauge theory side. In the Euclidean picture, we show explicitly that our bosonization map is equivalent to introducing the topological term in the action,

$$
S_{\text {top }}=i \pi \int_{Y}\left(A_{n-1} \cup_{n-3} A_{n-1}+A_{n-1} \cup_{n-2} \delta A_{n-1}\right) \text {, }
$$

where $A_{n-1}$ is a $(n-1)$-form gauge field, a $(n-1)$-cochain $A_{n-1} \in C^{n-1}\left(Y, \mathbb{Z}_{2}\right)$, and $Y$ is $(n+1)$-dimensional space-time manifold. When $A_{n-1}$ is closed, i.e., $\delta A=0$, this term reduces to the Steenrod square operator [4]. This Steenrod square term appears in the Lagrangian of fermionic symmetry-protected topological (SPT) phases [5] and it is indirectly argued that this term plays the role of statistical transmutation, which makes the theory fermionic [6,7]. Our approach provides an explicit Hamiltonian picture and the bosonization or fermionization procedure is exact, which gives the direct construction for supercohomology fermionic SPT phases. The quantum circuit for the supercohomology SPT ground state and its commuting projector Hamiltonian are derived explicitly in Ref. [8]. All supercohomology fermionic SPT phases in arbitrary dimensions can be constructed from the bosonization map presented in this paper.

There are already several proposals for an analog of the Jordan-Wigner map in arbitrary dimensions [9-12]. Our construction is most similar to that of Bravyi and Kitaev [9]. One advantage of our construction is that we can clearly identify the kind of $n$-dimensional bosonic systems that are dual to fermionic systems: They possess global $(n-1)$-form $\mathbb{Z}_{2}$ symmetry with a specific 't Hooft anomaly, as proposed in Ref. [3]. It is also manifest in our approach that the bosonization map depends on a choice of spin structure.

distribution of this work must maintain attribution to the author(s) and the published article's title, journal citation, and DOI. 


\section{CHAINS, COCHAINS, AND HIGHER CUP PRODUCTS}

In this section, we introduce the mathematical tools used in this paper. Our notations and conventions are also described. We will always work with an arbitrary triangulation of a closed simply connected $n$-dimensional manifold $M_{n}$ equipped with a branching structure (orientations on edges without forming a loop in any triangle). ${ }^{2}$ The vertices, edges, faces, and tetrahedra are denoted $v, e, f, t$, respectively. The general $d$ simplex is denoted as $\Delta_{d}$. We can label the vertices of $\Delta_{d}$ as $0,1,2, \ldots, d$ such that the directions of edges are from the small number to the larger number. We denote this $d$ simplex as $\Delta_{d}=\langle 01 \ldots d\rangle$. Its boundaries are $(d-1)$ simplices $\langle 0, \ldots, \hat{i}, \ldots, d\rangle$ for $i=0,1, \ldots, d$, where $\hat{i}$ means $i$ is omitted. A formal sum of $d$-simplices modulo 2 forms an element of the chain $C_{d}\left(M_{n}, \mathbb{Z}_{2}\right)$.

For every $v$, we define its dual 0 -cochain $\boldsymbol{v}$, which takes value 1 on $v$, and 0 otherwise, i.e., $\boldsymbol{v}\left(v^{\prime}\right)=\delta_{v, v^{\prime}}$. Similarly, $\mathbf{e}$ is an 1-cochain $\boldsymbol{e}\left(e^{\prime}\right)=\delta_{e, e^{\prime}}$, and so forth, i.e., $\boldsymbol{\Delta}_{d}$ being a $d$-cochain $\Delta_{d}\left(\Delta_{d}^{\prime}\right)=\delta_{\Delta_{d}, \Delta_{d}^{\prime}}$. All dual cochains will be denoted in bold. A $d$-cochain $c_{d} \in C^{d}\left(M_{n}, \mathbb{Z}_{2}\right)$ can be identified as a $\mathbb{Z}_{2}$ field living on each $d$-simplex $\Delta_{d}$, with the value $\boldsymbol{c}_{d}\left(\Delta_{d}\right)$. An evaluation of a cochain $c$ on a chain $c^{\prime}$ is the sum of $\boldsymbol{c}$ evaluated on simplices in $c^{\prime}$, which is denoted $\int_{c^{\prime}} \boldsymbol{c}=$ $\sum_{\Delta \in c^{\prime}} \boldsymbol{c}(\Delta)$, When the integration range is not written, $\boldsymbol{c}$ is assumed to be the top dimension and $\int \boldsymbol{c} \equiv \int_{M_{n}} \boldsymbol{c}$.

The boundary operator is denoted by $\partial$. For an $n$-simplex $\Delta_{n}, \partial \Delta_{n}$ consists of all boundary $(n-1)$-simplices of $\Delta_{n}$ :

$$
\partial(\langle 0,1,2, \ldots, d\rangle)=\sum_{i=0}^{d}\langle 0, \ldots, \hat{i}, \ldots, d\rangle .
$$

The coboundary operator is denoted by $\delta$ (not to be confused with the Kronecker delta previously). On a 0 -cochain $\boldsymbol{v}, \delta \boldsymbol{v}$ is an 1-cochain acting on edges, and is 1 if $\partial e$ contains $v$ and 0 otherwise:

$$
\delta \boldsymbol{v}(e)=\boldsymbol{v}(\partial e)=\delta_{v, \partial e} .
$$

It is similar for simplices in any dimension. For any $d$-cochain $c \in C^{d}\left(M_{n}, \mathbb{Z}_{2}\right)$, its coboundary $\delta c \in C^{d+1}\left(M_{n}, \mathbb{Z}_{2}\right)$ acting on a $(d+1)$-simplex $\Delta_{d+1}=\langle 0,1, \ldots, d+1\rangle$ is defined by

$$
\begin{aligned}
\delta \boldsymbol{c}\left(\Delta_{d+1}\right) & \equiv \boldsymbol{c}\left(\partial \Delta_{d+1}\right) \\
& =\sum_{i=0}^{d+1} \boldsymbol{c}(\langle 0, \ldots, \hat{i}, \ldots, d+1\rangle) .
\end{aligned}
$$

The cup product $\cup$ of a $p$-cochain $\alpha_{p}$ and a $q$-cochain $\beta_{q}$ is a $(p+q)$-cochain defined as

$$
\begin{aligned}
& {\left[\alpha_{p} \cup \beta_{q}\right](\langle 0,1, \ldots, p+q\rangle)} \\
& \quad=\alpha_{p}(\langle 01 \ldots p\rangle) \beta_{q}(\langle p, p+1, \ldots, p+q\rangle) \\
& \quad=\alpha_{p}(\langle 0 \sim p\rangle) \beta_{q}(\langle p \sim p+q\rangle),
\end{aligned}
$$

\footnotetext{
${ }^{2} \mathrm{~A}$ direct construction of branching structure is to arbitrarily assign different real numbers on all vertices. For each edge, the arrow is pointed from the smaller number to the larger number.
}

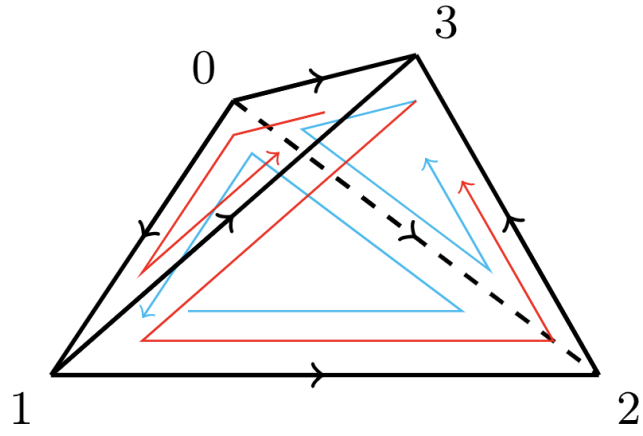

FIG. 1. A branching structure on a tetrahedron. The orientation of each face is determined by the right-hand rule. We defined this as the + tetrahedron, the directions of faces $\langle 012\rangle$ and $\langle 023\rangle$ are inward (blue) while the directions of faces $\langle 123\rangle$ and $\langle 013\rangle$ are outward (red). The directions of faces are reversed in the - tetrahedron (mirror image of this tetrahedron) [2].

where $i \sim j$ represents the integers from $i$ to $j$, i.e., $i, i+$ $1, \ldots, j$. The definition of the (higher) cup product $\cup_{1}[3,4]$ is

$$
\begin{aligned}
& {\left[\alpha_{p} \cup_{1} \beta_{q}\right](\langle 0, \cdots, p+q-1\rangle)} \\
& \quad=\sum_{i_{0}=0}^{p+q-1} \alpha_{p}\left(\left\langle 0 \sim i_{0}, i_{0}+q \sim p+q-1\right\rangle\right) \\
& \quad \times \beta_{q}\left(\left\langle i_{0} \sim i_{0}+q\right\rangle\right),
\end{aligned}
$$

and the next cup product $\cup_{2}$ is

$$
\begin{aligned}
& {\left[\alpha_{p} \cup_{2} \beta_{q}\right](\langle 0, \cdots, p+q-2\rangle)} \\
& \quad=\sum_{0 \leqslant i_{0}<i_{1} \leqslant p+q-2} \alpha_{p}\left(\left\langle 0 \sim i_{0}, i_{1} \sim p+i_{1}-i_{0}-1\right\rangle\right) \\
& \quad \times \beta_{q}\left(\left\langle i_{0} \sim i_{1}, p+i_{1}-i_{0}-1 \sim p+q-2\right\rangle\right) .
\end{aligned}
$$

The general higher cup product can be expressed as

$$
\begin{aligned}
& {\left[\alpha_{p} \cup_{a} \beta_{q}\right](0,1, \cdots, p+q-a)} \\
& \quad=\sum_{0 \leqslant i_{0}<i_{1}<\cdots<i_{a} \leqslant p+q-a} \alpha_{p}\left(\left\langle 0 \sim i_{0}, i_{1} \sim i_{2}, i_{3} \sim i_{4}, \cdots\right\rangle\right) \\
& \quad \times \beta_{q}\left(\left\langle i_{0} \sim i_{1}, i_{2} \sim i_{3}, \cdots\right\rangle\right),
\end{aligned}
$$

where $\left\{i_{0}, i_{1}, \ldots, i_{a}\right\}$ are chosen such that the arguments of $\alpha_{p}$ and $\beta_{q}$ contain $p+1$ and $q+1$ numbers separately. For example, we have

$$
\alpha_{2} \cup_{1} \beta_{1}(\langle 012\rangle)=\alpha_{2}(\langle 012\rangle) \beta_{1}(\langle 01\rangle)+\alpha_{2}(\langle 012\rangle) \beta_{1}(\langle 12\rangle),
$$

since the allowed choices are only $\left(i_{0}, i_{1}\right)=(0,1)$ and $\left(i_{0}, i_{1}\right)=(1,2)$. Another example is

$$
\begin{aligned}
\alpha_{2} & \cup_{1} \beta_{2}(\langle 0123\rangle) \\
& =\alpha_{2}(\langle 023\rangle) \beta_{2}(\langle 012\rangle)+\alpha_{2}(\langle 013\rangle) \beta_{2}(\langle 123\rangle),
\end{aligned}
$$

where the choices of $\left(i_{0}, i_{1}\right)$ are $(0,2)$ and $(1,3)$. Notice that faces $\langle 023\rangle$ and $\langle 012\rangle$ are inward, while faces $\langle 013\rangle$ and $\langle 123\rangle$ are outward in Fig. 1. Therefore, the $\cup_{1}$ product of two 2cochains acting on a tetrahedron is the sum of the products of 2-cochains acting faces with the same orientation (either both inward or both outward). In Sec. IV A, this property can be 


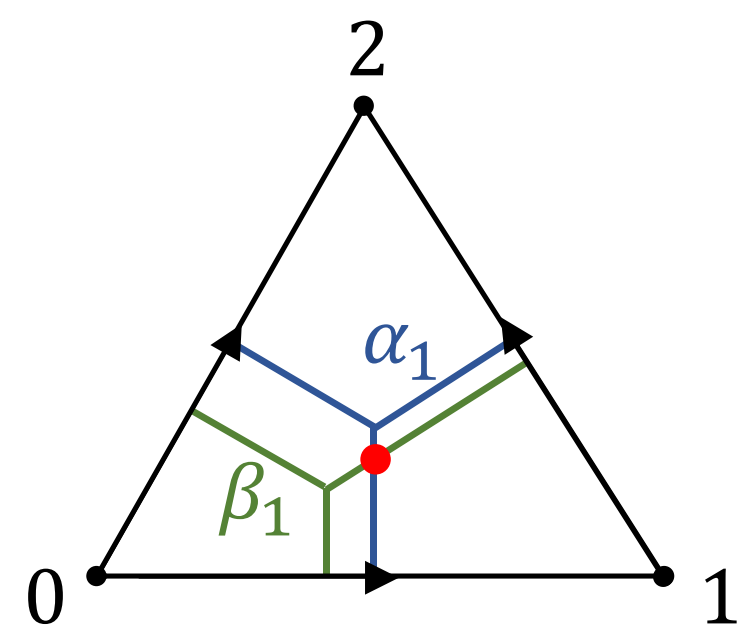

FIG. 2. In this figure, $\alpha_{1}$ is represented by blue segments, dual (attached) to edges $\langle 01\rangle,\langle 12\rangle$, and $\langle 02\rangle$ separately. Similarly, $\beta_{1}$ is represented by green segments with a shifting. The red point is the intersection between the blue segment dual to edge $\langle 01\rangle$ and the green segment dual to edge $\langle 12\rangle$. Therefore, we can read that the cup product of $\alpha_{1}$ and $\beta_{1}$ acting on this triangle $\langle 012\rangle$ is $\alpha_{1}(\langle 01\rangle) \beta_{1}(\langle 12\rangle)$.

generalized to higher dimensions: the $\cup_{n-2}$ of two $(n-1)$ cochains acting on an $n$-simplex is the sum of the product of $(n-1)$-cochains acting on its boundary $(n-1)$-simplices with the same orientation. This geometry interpretation of higher cup products is crucial since it is further shown that this property coincides with the commutation relations of fermionic hopping operators. The fermionic statistic is captured by higher cup products and this makes it convenient to derive the topological action for fermionic theories. Although not directly used in this paper, a higher cup product of arbitrary cochains has a nice geometrical interpretation [13]: The higher cup product measures the intersection between dual cells and thickened, shifted version of other dual cells, where the thickening and shifting are determined by the vector frame field. For example, the simplest cup product formula

$$
\alpha_{1} \cup \beta_{1}(\langle 012\rangle)=\alpha_{1}(\langle 01\rangle) \beta_{1}(\langle 12\rangle),
$$

can be viewed as the intersection point in Fig. 2.

It should be emphasized that the cup products satisfy the recursive property

$$
\alpha \cup_{a} \beta+\beta \cup_{a} \alpha=\alpha \cup_{a+1} \delta \beta+\delta \alpha \cup_{a+1} \beta+\delta\left(\alpha \cup_{a+1} \beta\right),
$$

which can be interpreted as that the noncommutative property of the $\cup_{a}$ product is equal to the failure of the product rule of the coboundary operation $\delta$ on the $\cup_{a+1}$ product.

Finally, $\Delta_{n}^{1} \supset \Delta_{n^{\prime}}^{2}$ or $\Delta_{n^{\prime}}^{2} \subset \Delta_{n}^{1}$ means that the simplex $\Delta_{n}^{1}$ contains $\Delta_{n^{\prime}}^{2}$ as a subsimplex. A general rule of thumb is that the subset symbol always points to one higher dimension.

\section{REVIEW OF BOSON-FERMION DUALITY IN (2+1)D AND (3+1)D}

We begin by reviewing the duality between fermions and $\mathbb{Z}_{2}$ lattice gauge theory in both two spatial dimensions [1] and three spatial dimensions [2]. On each face $f$ of the 2-manifold $M_{2}$, we place a single pair of fermionic creation-annihilation operators $c_{f}, c_{f}^{\dagger}$, or equivalently a pair of Majorana fermions:

$$
\gamma_{f}=c_{f}+c_{f}^{\dagger}, \quad \gamma_{f}^{\prime}=\frac{c_{f}-c_{f}^{\dagger}}{i} .
$$

The algebra of Majorana fermions is

$$
\left\{\gamma_{f}, \gamma_{f^{\prime}}\right\}=\left\{\gamma_{f}^{\prime}, \gamma_{f^{\prime}}^{\prime}\right\}=2 \delta_{f, f^{\prime}}, \quad\left\{\gamma_{f}, \gamma_{f^{\prime}}^{\prime}\right\}=0,
$$

where $\{A, B\}=A B-B A$ is the anticommutator. The even fermionic algebra consists of local observables with a trivial fermionic parity (i.e., $P_{F}=\prod_{f}(-1)^{c_{f}^{\dagger} c_{f}}=1$ ). It is generated by the on-site fermion parity,

$$
P_{f}=-i \gamma_{f} \gamma_{f}^{\prime},
$$

and the fermionic hopping operator on every edge $e$,

$$
S_{e}=i \gamma_{L(e)} \gamma_{R(e)}^{\prime},
$$

where $L(e)$ and $R(e)$ are faces to the left and right of $e$, with respect to the branching structure of $e$. The commutation relation of hopping operators can be expressed as

$$
S_{e} S_{e^{\prime}}=(-1)^{\int e \cup e^{\prime}+e^{\prime} \cup e} S_{e} S_{e^{\prime}},
$$

where the sign from the commutation occurs only when the arrows on the two edges follow head to tail and are on the same triangle, i.e., edges $\left\{e, e^{\prime}\right\}$ being $\{\langle 01\rangle,\langle 12\rangle\}$ of a triangle $\langle 012\rangle$. In general, for any 1 -cochains $\lambda$ and $\lambda^{\prime}$,

$$
S_{\lambda+\lambda^{\prime}} \equiv(-1)^{\int \lambda \cup \lambda^{\prime}} S_{\lambda^{\prime}} S_{\lambda} .
$$

In other words, $S_{\lambda}$ is the product of $S_{e}$ over $\{e \mid \lambda(e)=1\}$ and the sign in front is consistent with the commutation relations. If we consider the product of fermionic hopping operators on edges around a vertex $v$, the Majorana operators cancel out up to some $P_{f}$ terms. The two generators $P_{f}$ and $S_{e}$ satisfy the following constraint at each vertex $v[1]$ :

$$
(-1)^{\int_{w_{2}} v} S_{\delta v} \prod_{f} P_{f}^{\int v \cup f+f \cup v}=1,
$$

where $w_{2} \in C_{0}\left(M_{2}, \mathbb{Z}_{2}\right)$ is the 0 -chain which is Poincaré dual to the second Stiefel-Whitney cohomology class $\boldsymbol{w}_{2}\left(M_{2}\right)$. The explicit expression of $w_{2}$ is given in the Appendix. We require $M_{2}$ to be a spin manifold, i.e., the second Stiefel-Whitney class is exact: $w_{2}=\partial E$ for some $E \in C_{1}\left(M_{2}, \mathbb{Z}_{2}\right)$. The 1chain $E$ is a choice of the spin structure. The nonexactness of the second Stiefel-Whitney class is the obstruction to determine this 1-chain $E$, which prevents us from defining a self-consistent bosonization map, which dualizes the even sector of fermionic Hilbert space to a $\mathbb{Z}_{2}$ gauge theory.

The bosonic dual of this system involves $\mathbb{Z}_{2}$-valued spins on the edges of the triangulation. The bosonic algebra are generated by Pauli matrix on edges:

$$
X_{e}=\left[\begin{array}{ll}
0 & 1 \\
1 & 0
\end{array}\right], \quad Y_{e}=\left[\begin{array}{cc}
0 & -i \\
i & 0
\end{array}\right], \quad Z_{e}=\left[\begin{array}{cc}
1 & 0 \\
0 & -1
\end{array}\right] .
$$

For every face $f$, we define the flux operator,

$$
W_{f}=\prod_{e \subset f} Z_{e},
$$


and for every edge $e$ we define a unitary operator $U_{e}$ which squares to 1:

$$
U_{e}=X_{e}\left(\prod_{e^{\prime}} Z_{e^{\prime}}^{\int e^{\prime} \cup e}\right),
$$

where $X_{e}, Z_{e}$ are Pauli matrices acting on a spin at the edge $e$. It has been shown in Ref. [1] that the sets $\left\{U_{e}, W_{f}\right\}$ and $\left\{S_{e}, P_{f}\right\}$ satisfy the same commutation relations. The boson-fermion duality map defined on the manifold $M_{2}$ is

$$
\begin{aligned}
W_{f}= & \prod_{e \subset f} Z_{e} \longleftrightarrow P_{f}=-i \gamma_{f} \gamma_{f}^{\prime}, \\
U_{e}= & X_{e}\left(\prod_{e^{\prime}} Z_{e^{\prime}}^{\int e^{\prime} \cup e}\right) \longleftrightarrow(-1)^{\int_{E} e} S_{e} \\
= & (-1)^{\int_{E}} \boldsymbol{e}_{i \gamma_{L(e)} \gamma_{R(e)}^{\prime},} \\
G_{v}= & \prod_{e \supset v} X_{e}\left(\prod_{e^{\prime}} Z_{e^{\prime}}^{\int \delta v \cup \boldsymbol{e}^{\prime}}\right) \longleftrightarrow(-1)^{\int_{w_{2}}}{ }^{v} S_{\delta \boldsymbol{v}} \\
& \times \prod_{f} P_{f}^{\int v \cup f+f \cup v} \\
= & 1, \\
\prod_{f} W_{f}= & 1 \longleftrightarrow \prod_{f} P_{f},
\end{aligned}
$$

where the 0-chain $w_{2} \in C_{0}\left(M_{2}, \mathbb{Z}_{2}\right)$ is the chain representation of the second Stiefel-Whitney class and the 1-chain $E \in C_{1}\left(M_{2}, \mathbb{Z}_{2}\right)$ denotes a choice of spin structure $\left(\partial E=w_{2}\right)$. For the consistency of this duality map, we need to impose the gauge constraints on bosonic side $\prod_{e \supset v} X_{e}\left(\prod_{e^{\prime}} Z_{e^{\prime}}^{\int \delta v \cup \boldsymbol{e}^{\prime}}\right)=1$. The gauge invariant subspace in the bosonic Hilbert space is dual to the fermionic system with total fermion parity $\prod_{f} P_{f}=1$.

The 3D boson-fermion duality defined on a 3D manifold $M_{3}$ can be done in a similar way [2]. The only difference is that the fermions $\gamma_{t}, \gamma_{t}^{\prime}$ are at the center of tetrahedra $t$ and Pauli operators $X_{f}, Z_{f}$ live on faces $f$. In three spatial dimensions, any fermionic system can be mapped to a 2 -form $\mathbb{Z}_{2}$ gauge theory on the 3D lattice. The duality dictionary becomes

$$
\begin{aligned}
W_{t}= & \prod_{f \subset t} Z_{f} \longleftrightarrow P_{t}=-i \gamma_{t} \gamma_{t}^{\prime}, \\
U_{f}= & X_{f}\left(\prod_{f^{\prime}} Z_{f^{\prime}}^{\int f^{\prime} \cup_{1} f}\right) \longleftrightarrow(-1)^{\int_{E}} f_{S_{f}} \\
= & (-1)^{\int_{E}} f_{i \gamma_{L}(f)} \gamma_{R(f)}^{\prime}, \\
G_{e}= & \prod_{f \supset e} X_{f}\left(\prod_{f^{\prime}} Z_{f^{\prime}}^{\int \delta e \cup_{1} f^{\prime}}\right) \longleftrightarrow(-1)^{\int_{w_{2}}}{ }^{e} S_{\delta e} \\
& \times \prod_{t} P_{t}^{\int e \cup_{1} t+t \cup_{1} e}=1, \\
\prod_{t} W_{t}= & 1 \longleftrightarrow \prod_{t} P_{t},
\end{aligned}
$$

where the 1-chain $w_{2} \in C_{1}\left(M_{3}, \mathbb{Z}_{2}\right)$ is the chain representative of the second Stiefel-Whitney class and the 2-chain $E \in C_{2}\left(M_{3}, \mathbb{Z}_{2}\right)$ is a choice of spin structure $\left(\partial E=w_{2}\right)$.

\section{EXACT BOSONIZATION IN $\boldsymbol{n}$ DIMENSIONS}

From the 2D and 3D formulas Eqs. (21) and (22), it is very natural to conjecture the $n$-dimensional boson-fermion duality. Consider a spin manifold $M_{n}$ in spatial $n$ dimensions. The fermions live at the center $n$-simplices, i.e., $\gamma_{\Delta_{n}}, \gamma_{\Delta_{n}}^{\prime}$ for each $\Delta_{n}$. The Pauli matrices live on $(n-1)$-simplices, i.e., $X_{\Delta_{n-1}}$ and $Z_{\Delta_{n-1}}$ for each $\Delta_{n-1}$. The $n$-dimensional boson-fermion duality should be

$$
\begin{aligned}
& W_{\Delta_{n}} \equiv \prod_{\Delta_{n-1} \subset \Delta_{n}} Z_{\Delta_{n-1}} \longleftrightarrow P_{t}=-i \gamma_{\Delta_{n}} \gamma_{\Delta_{n}}^{\prime}, \\
& U_{\Delta_{n-1}} \equiv X_{\Delta_{n-1}}\left(\prod_{\Delta_{n-1}{ }^{\prime}} Z_{\Delta_{n-1}{ }^{\prime}}^{\int \Delta_{n-1} \cup_{n-2} \Delta_{n-1}}\right) \longleftrightarrow(-1)^{\int_{E} \Delta_{n-1}} S_{\Delta_{n-1}} \\
& =(-1)^{\int_{E} \Delta_{n-1}} i \gamma_{L\left(\Delta_{n-1}\right)} \gamma_{R\left(\Delta_{n-1}\right)}^{\prime} \text {, } \\
& G_{\Delta_{n-2}} \equiv \prod_{\Delta_{n-1} \supset \Delta_{n-2}} X_{\Delta_{n-1}}\left(\prod_{\Delta_{n-1}{ }^{\prime}} Z_{\Delta_{n-1}}^{\int \delta \boldsymbol{\Delta}_{n-2} \cup_{n-2} \boldsymbol{\Delta}_{n-1}{ }^{\prime}}\right) \\
& \longleftrightarrow(-1)^{\int_{w_{2}} \Delta_{n-2}} S_{\delta \Delta_{n-2}} \\
& \times \prod_{\Delta_{n}} P_{\Delta_{n}}^{\int \Delta_{n-2} \cup_{n-2} \Delta_{n}+\Delta_{n} \cup_{n-2} \Delta_{n-2}}=1, \\
& \prod_{\Delta_{n}} W_{\Delta_{n}}=1 \longleftrightarrow \prod_{\Delta_{n}} P_{\Delta_{n}},
\end{aligned}
$$

where $w_{2} \in C_{n-2}\left(M_{n}, \mathbb{Z}_{2}\right)$ is the chain representative of the second Stiefel-Whitney class, $E \in C_{n-1}\left(M_{n}, \mathbb{Z}_{2}\right)$ denotes a choice of spin structure $\left(\partial E=w_{2}\right)$, and for general $(n-1)$ cochain $\lambda_{n-1}$ and $\lambda_{n-1}^{\prime}$, the product of $S$ operators is defined as

$$
S_{\lambda_{n-1}+\lambda_{n-1}^{\prime}} \equiv(-1)^{\int \lambda_{n-1} \cup_{n-2} \lambda_{n-1}^{\prime}} S_{\lambda_{n-1}^{\prime}} S_{\lambda_{n-1}} .
$$

This $n$-dimensional boson-fermion duality Eq. (23) is the main theorem of this paper, which will be proved by the end of this section.

\section{A. Commutation relations}

Consider an $n$-simplex $\Delta_{n}=\langle 012 \ldots n\rangle$. Its boundary contains all $(n-1)$-simplex $\left\langle\partial \Delta_{n}\right\rangle^{i}=\langle 0 \ldots \hat{i} \ldots n\rangle$ where $\hat{i}$ means the vertex $i$ is omitted. We define the orientation of $\left\langle\partial \Delta_{n}\right\rangle^{i}$ as $O\left(\left\langle\partial \Delta_{n}\right\rangle^{i}\right)=(-1)^{i}$. For +-oriented $\Delta_{n}$, if $O\left(\left\langle\partial \Delta_{n}\right\rangle^{i}\right)=1$, the boundary $\left\langle\partial \Delta_{n}\right\rangle^{i}$ is outward, and if $O\left(\left\langle\partial \Delta_{n}\right\rangle^{i}\right)=-1$, the boundary $\left\langle\partial \Delta_{n}\right\rangle^{i}$ is inward. For oriented $\Delta_{n}$, the inward and outward boundaries are opposite. $S_{\Delta_{n-1}}$ and $S_{\Delta_{n-1}^{\prime}}$ anticommute only when $\Delta_{n-1}$ and $\Delta_{n-1}^{\prime}$ are both inward or both outward boundaries of some $n$-simplex, i.e., $\Delta_{n-1}, \Delta_{n-1}^{\prime} \in \partial \Delta_{n}$. We are going to prove that this is equivalent to

$S_{\Delta_{n-1}} S_{\Delta_{n-1}^{\prime}}=(-1)^{\int \Delta_{n-1} \cup_{n-2} \Delta_{n-1}^{\prime}+\Delta_{n-1}^{\prime} \cup_{n-2} \Delta_{n-1}} S_{\Delta_{n-1}^{\prime}} S_{\Delta_{n-1}}$. 
(a)

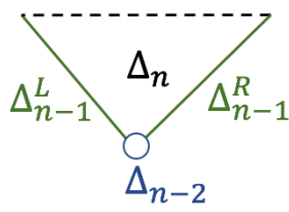

(d)

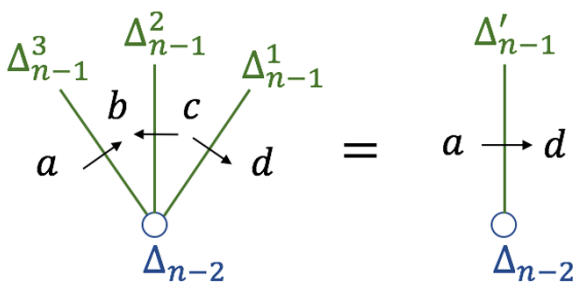

(b)

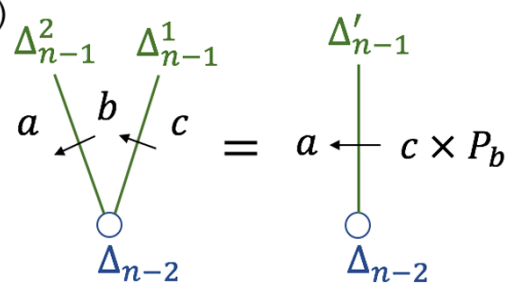

(c)

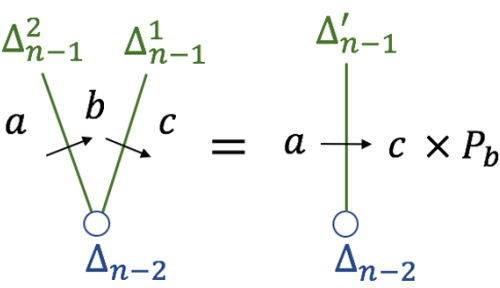

FIG. 3. (a) The $n$-simplex $\Delta_{n}$ is formed by the $(n-1)$-simplex $\Delta_{n-2}$ and two $(n-1)$-simplex $\Delta_{n-1}^{L}$ and $\Delta_{n-1}^{R}$. (b) The product of $S_{\Delta_{n-2}}$ is $\left(i \gamma_{b} \gamma_{a}^{\prime}\right)\left(i \gamma_{c} \gamma_{b}^{\prime}\right)=\left(i \gamma_{c} \gamma_{a}^{\prime}\right)\left(-i \gamma_{b} \gamma_{b}^{\prime}\right)=\left(i \gamma_{c} \gamma_{a}^{\prime}\right) P_{b}$. (c) The product of $S_{\Delta_{n-2}}$ is $\left(i \gamma_{a} \gamma_{b}^{\prime}\right)\left(i \gamma_{b} \gamma_{c}^{\prime}\right)=\left(i \gamma_{a} \gamma_{c}^{\prime}\right)\left(-i \gamma_{b} \gamma_{b}^{\prime}\right)=\left(i \gamma_{a} \gamma_{c}^{\prime}\right) P_{b}$. (d) The product of $S_{\Delta_{n-2}}$ is $\left(i \gamma_{a} \gamma_{b}^{\prime}\right)\left(i \gamma_{c} \gamma_{b}^{\prime}\right)\left(i \gamma_{c} \gamma_{d}^{\prime}\right)=i \gamma_{a} \gamma_{d}^{\prime}$. (e) The product of $S_{\Delta_{n-2}}$ is $\left(i \gamma_{b} \gamma_{a}^{\prime}\right)\left(i \gamma_{b} \gamma_{c}^{\prime}\right)\left(i \gamma_{d} \gamma_{c}^{\prime}\right)=i \gamma_{d} \gamma_{a}^{\prime}$.

From the definition of the higher cup product Eq. (8), we have

$$
\begin{aligned}
{\left[\boldsymbol{\Delta}_{n-1} \cup_{n-2} \boldsymbol{\Delta}_{n-1}^{\prime}\right](0,1, \ldots, n)=} & \sum_{0 \leqslant i_{0}<i_{1}<\cdots<i_{n-2} \leqslant n} \boldsymbol{\Delta}_{n-\mathbf{1}}\left(0 \sim i_{0}, i_{1} \sim i_{2}, i_{3} \sim i_{4}, \ldots\right) \boldsymbol{\Delta}_{n-1}^{\prime}\left(i_{0} \sim i_{1}, i_{2} \sim i_{3}, \ldots\right) \\
= & \sum_{0 \leqslant j_{1}<j_{2} \leqslant n \mid j_{1}, j_{2} \in \text { even }} \boldsymbol{\Delta}_{n-1}\left(\left\langle 0 \ldots \hat{j_{2}} \ldots n\right\rangle\right) \boldsymbol{\Delta}_{n-1}^{\prime}\left(\left\langle 0 \ldots \hat{j_{1}} \ldots n\right\rangle\right) \\
& +\sum_{0 \leqslant k_{1}<k_{2} \leqslant n \mid k_{1}, k_{2} \in \text { odd }} \boldsymbol{\Delta}_{n-1}\left(\left\langle 0 \ldots \hat{k_{1}} \ldots n\right\rangle\right) \boldsymbol{\Delta}_{n-1}^{\prime}\left(\left\langle 0 \ldots \hat{k_{2}} \ldots n\right\rangle\right) .
\end{aligned}
$$

The $\cup_{n-2}$ only contains the product of boundaries $\Delta_{n-1}^{i}$ with the same orientation (inward or outward) and each pair of $\Delta_{n-1}^{i}, \Delta_{n-1}^{i^{\prime}}$ with the same orientation appears exactly once. Therefore, the $\cup_{n-2}$ expression in Eq. (25) captures the commutation relations of fermionic hopping operators $S_{\Delta_{n-1}}$. It is easy to check that bosonic operators $U_{\Delta_{n-1}}$ satisfy the same commutation relations:

$$
U_{\Delta_{n-1}} U_{\Delta_{n-1}^{\prime}}=(-1)^{\int \Delta_{n-1} \cup_{n-2} \Delta_{n-1}^{\prime}+\Delta_{n-1}^{\prime} \cup_{n-2} \Delta_{n-1}} U_{\Delta_{n-1}^{\prime}} U_{\Delta_{n-1}} .
$$

Therefore, $\left\{S_{\Delta_{n-1}}, P_{\Delta_{n}}\right\}$ and $\left\{U_{\Delta_{n-1}}, W_{\Delta_{n}}\right\}$ in Eq. (23) have the same commutation relations.

\section{B. Gauge constraints}

In this section, we will derive the constraints on fermionic operators:

$$
(-1)^{\int_{w_{2}} \boldsymbol{\Delta}_{n-2}} S_{\delta \Delta_{n-2}} \prod_{\Delta_{n}} P_{\Delta_{n}}^{\int \boldsymbol{\Delta}_{n-2} \cup_{n-2} \boldsymbol{\Delta}_{n}+\boldsymbol{\Delta}_{n} \cup_{n-2} \boldsymbol{\Delta}_{n-2}}=1 .
$$

This follows directly from the following two lemmas.

Lemma 1. The Majorana operators in $S_{\delta \Delta_{n-2}}$ cancel out with Majorana operators in $\prod_{\Delta_{n}} P_{\Delta_{n}}^{\int \boldsymbol{\Delta}_{n-2} \cup_{n-2} \boldsymbol{\Delta}_{n}+\boldsymbol{\Delta}_{n} \cup_{n-2} \boldsymbol{\Delta}_{n-2}}$.
Lemma 2. The sign difference of $S_{\delta \Delta_{n-2}}$ and the product of on-site fermion parities $\prod_{\Delta_{n}} P_{\Delta_{n}}^{\int \boldsymbol{\Delta}_{n-2} \cup_{n-2} \boldsymbol{\Delta}_{n}+\boldsymbol{\Delta}_{n} \cup_{n-2} \boldsymbol{\Delta}_{n-2}}$ is $-(-1)^{\sum_{i=1}^{d} \int \Delta_{n-1}^{i-1} \cup_{n-2} \Delta_{n-1}^{i}}$ where we order $(n-1)$ simplices $\quad\left\{\Delta_{n-1} \mid \Delta_{n-1} \supset \Delta_{n-2}\right\}$ counterclockwise as $\boldsymbol{\Delta}_{n-1}^{1}, \boldsymbol{\Delta}_{n-1}^{2}, \ldots, \boldsymbol{\Delta}_{n-1}^{d-1}, \boldsymbol{\Delta}_{n-1}^{d} \equiv \boldsymbol{\Delta}_{n-1}^{0}$, as shown in Fig. 4. This sign is a chain representative of the second Stiefel-Whitney class:

$$
-(-1)^{\sum_{i=1}^{d} \int \Delta_{n-1}^{i-1} \cup_{n-2} \Delta_{n-1}^{i}}=(-1)^{\int_{w_{2}} \Delta_{n-2}} .
$$

Proof of Lemma 1. Let us denote $\Delta_{n}=\langle 01 \ldots n\rangle$ formed by $\Delta_{n-2}$ and two $(n-1)$-simplex $\Delta_{n-1}^{L}$ and $\Delta_{n-1}^{R}$, shown in Fig. 3(a). We know that $S_{\delta \Delta_{n-2}}$ contains $\gamma_{\Delta_{n}} \gamma_{\Delta_{n}}^{\prime}$ if and only if $\Delta_{n-1}^{L}, \Delta_{n-1}^{R}$ are one inward boundary and one outward boundary of $n$-simplex $\Delta_{n}$, as indicated in Figs. 3(b) and $3(\mathrm{c})$.

For the product of $P_{\Delta_{n}}$, we simplify the integral as

$$
\int \boldsymbol{\Delta}_{n-2} \cup_{n-2} \boldsymbol{\Delta}_{n}+\boldsymbol{\Delta}_{n} \cup_{n-2} \boldsymbol{\Delta}_{n-2}=\int \delta \boldsymbol{\Delta}_{n-2} \cup_{n-1} \boldsymbol{\Delta}_{n},
$$

where we have used the property $\delta\left(\alpha \cup_{n-1} \beta\right)=\delta \alpha \cup_{n-1}$ $\beta+\alpha \cup_{n-1} \delta \beta+\alpha \cup_{n-2} \beta+\beta \cup_{n-2} \alpha$ and $\delta \Delta_{n}=0$ (since $n$ is the top dimension). The integral Eq. (30) has only the 
contribution from $\Delta_{n}=\langle 01 \ldots n\rangle$ :

$$
\begin{aligned}
\int \boldsymbol{\Delta}_{n-2} \cup_{n-2} \boldsymbol{\Delta}_{n}+\boldsymbol{\Delta}_{n} \cup_{n-2} \boldsymbol{\Delta}_{n-2} & =\left[\left(\boldsymbol{\Delta}_{n-1}^{L}+\boldsymbol{\Delta}_{n-1}^{R}\right) \cup_{n-1} \boldsymbol{\Delta}_{n}\right](\langle 01 \ldots n\rangle) \\
& =\sum_{0 \leqslant i_{0}<i_{1}<\cdots<i_{n-1} \leqslant n}\left(\boldsymbol{\Delta}_{n-1}^{L}+\boldsymbol{\Delta}_{n-1}^{R}\right)\left(0 \sim i_{0}, i_{1} \sim i_{2}, i_{3} \sim i_{4}, \ldots\right) \boldsymbol{\Delta}_{n}\left(i_{0} \sim i_{1}, i_{2} \sim i_{3}, \ldots\right) \\
& =\sum_{0 \leqslant j \leqslant n \mid j \in \text { odd }}\left(\Delta_{n-1}^{L}+\Delta_{n-1}^{R}\right)(\langle 0 \ldots \hat{j} \ldots n\rangle) \Delta_{n}(\langle 01 \ldots n\rangle) \\
& =\sum_{0 \leqslant j \leqslant n \mid j \in \text { odd }}\left(\Delta_{n-1}^{L}+\Delta_{n-1}^{R}\right)(\langle 0 \ldots \hat{j} \ldots n\rangle),
\end{aligned}
$$

which is 1 if and only $\Delta_{n-1}^{L}, \Delta_{n-1}^{R}$ are one inward boundary and one outward boundary of the $n$-simplex $\Delta_{n}$. This shows that product of $P_{\Delta_{n}}$ contain $P_{\Delta_{n}} \sim \gamma_{\Delta_{n}} \gamma_{\Delta_{n}}^{\prime}$ if and only if $\Delta_{n-1}^{L}, \Delta_{n-1}^{R}$ are one inward boundary and one outward boundary of the $n$-simplex $\Delta_{n}$. This cancels out with $S_{\delta \Delta_{n-2}}$ exactly.

Proof of Lemma 2. We compare the signs between

$$
\begin{aligned}
S_{\delta \Delta_{n-2}}= & (-1)^{\sum_{\Delta_{n-1}<\Delta_{n-1}^{\prime} \mid \Delta_{n-1}, \Delta_{n-1}^{\prime} \supset \Delta_{n-2}} \Delta_{n-1} \cup_{n-2} \Delta_{n-1}^{\prime}} \\
& \times \prod_{\Delta_{n-1} \supset \Delta_{n-2}} S_{\Delta_{n-1}}
\end{aligned}
$$

and

$$
\prod_{\Delta_{n}} P_{\Delta_{n}}^{\int \boldsymbol{\Delta}_{n-2} \cup_{n-2} \boldsymbol{\Delta}_{n}+\boldsymbol{\Delta}_{n} \cup_{n-2} \boldsymbol{\Delta}_{n-2},}
$$

where we have used the definition of $S_{\lambda_{n-1}}$ in Eq. (24). As shown in Fig. 4,

$$
\begin{aligned}
& S_{\Delta_{n-1}^{d}} \cdots S_{\Delta_{n-1}^{2}} S_{\Delta_{n-1}^{1}} \\
& \quad=S_{\Delta_{n-1}^{d}} S_{\Delta_{n-1}^{1}} \prod_{\Delta_{n} \neq a, b} P_{\Delta_{n}}^{\int \boldsymbol{\Delta}_{n-2} \cup_{n-2} \boldsymbol{\Delta}_{n}+\boldsymbol{\Delta}_{n} \cup_{n-2} \boldsymbol{\Delta}_{n-2} .}
\end{aligned}
$$

We can check that

$$
\begin{aligned}
S_{\Delta_{n-1}^{d}} S_{\Delta_{n-1}^{1}}= & -(-1)^{\int \boldsymbol{\Delta}_{n-1}^{1} \cup_{n-2} \boldsymbol{\Delta}_{n-1}^{d}+\boldsymbol{\Delta}_{n-1}^{d} \cup_{n-2} \boldsymbol{\Delta}_{n-1}^{1}} \\
& \times \prod_{\Delta_{n}=a, b} P_{\Delta_{n}}^{\int \boldsymbol{\Delta}_{n-2} \cup_{n-2} \boldsymbol{\Delta}_{n}+\boldsymbol{\Delta}_{n} \cup_{n-2} \boldsymbol{\Delta}_{n-2},},
\end{aligned}
$$

since $S_{\Delta_{n-1}^{d}} S_{\Delta_{n-1}^{1}}$ is 1 (or $-P_{a} P_{b}$ ) if $\Delta_{n-1}^{1}, \Delta_{n-1}^{d}$ are both inward or outward (or one inward and one outward) in $\Delta_{n}=a$. Therefore,

$$
\begin{aligned}
S_{\Delta_{n-1}^{d}} \cdots S_{\Delta_{n-1}^{2}} S_{\Delta_{n-1}^{1}}= & -(-1)^{\int \boldsymbol{\Delta}_{n-1}^{1} \cup_{n-2} \Delta_{n-1}^{d}+\boldsymbol{\Delta}_{n-1}^{d} \cup_{n-2} \boldsymbol{\Delta}_{n-1}^{1}} \\
& \times \prod_{\Delta_{n}} P_{\Delta_{n}}^{\int \boldsymbol{\Delta}_{n-2} \cup_{n-2} \boldsymbol{\Delta}_{n}+\boldsymbol{\Delta}_{n} \cup_{n-2} \boldsymbol{\Delta}_{n-2}} .
\end{aligned}
$$

Together with Eq. (32), we have

$$
\begin{aligned}
S_{\delta \Delta_{n-2}} & \prod_{\Delta_{n}} P_{\Delta_{n}}^{\int \boldsymbol{\Delta}_{n-2} \cup_{n-2} \boldsymbol{\Delta}_{n}+\boldsymbol{\Delta}_{n} \cup_{n-2} \boldsymbol{\Delta}_{n-2}} \\
= & (-1)^{\int \boldsymbol{\Delta}_{n-1}^{1} \cup_{n-2} \boldsymbol{\Delta}_{n-1}^{d}+\sum_{i=2}^{d} \int \boldsymbol{\Delta}_{n-1}^{i-1} \cup_{n-2} \boldsymbol{\Delta}_{n-1}^{i}} \\
& \times\left(-(-1)^{\int \boldsymbol{\Delta}_{n-1}^{1} \cup_{n-2} \boldsymbol{\Delta}_{n-1}^{d}+\boldsymbol{\Delta}_{n-1}^{d} \cup_{n-2} \boldsymbol{\Delta}_{n-1}^{1}}\right) \\
= & -(-1)^{\sum_{i=1}^{d} \int \boldsymbol{\Delta}_{n-1}^{i-1} \cup_{n-2} \boldsymbol{\Delta}_{n-1}^{i}} .
\end{aligned}
$$

From the definition of $\cup_{n-2}$ product Eq. (26),

$$
\begin{aligned}
& \sum_{i=1}^{d} \int \boldsymbol{\Delta}_{n-1}^{i-1} \cup_{n-2} \boldsymbol{\Delta}_{n-1}^{i} \\
& =\sum_{i=1}^{d} \sum_{\Delta_{n}} \boldsymbol{\Delta}_{n-1}^{i-1} \cup_{n-2} \boldsymbol{\Delta}_{n-1}^{i}\left(\Delta_{n}\right) \\
& =\sum_{- \text {oriented } \Delta_{n}=\langle 0 \ldots n\rangle j_{1}<j_{2} \mid j_{1}, j_{2} \in \text { even }} \boldsymbol{\Delta}_{n-2}\left(\left\langle 0 \cdots \hat{j_{1}} \cdots \hat{j}_{2} \cdots n\right\rangle\right) \\
& \quad+\sum_{\quad+\text { oriented } \Delta_{n}=\langle 0 \ldots n\rangle k_{1}<k_{2} \mid k_{1}, k_{2} \in \text { odd }} \\
& \quad \times \boldsymbol{\Delta}_{n-2}\left(\left\langle 0 \cdots \hat{k_{1}} \cdots \hat{k_{2}} \cdots n\right\rangle\right),
\end{aligned}
$$

where the relation between $\Delta_{n-2}, \Delta_{n-1}^{i}$, and $\Delta_{n}$ is shown in Fig. 4. The distinct orientations of --oriented $\Delta_{n}$ and + -oriented $\Delta_{n}$ in the summation come from the fact that $j_{1}, j_{2}$ and $k_{1}, k_{2}$ in Eq. (26) have opposite orders. Equation (38) is related to $w_{2}$ by the following Lemma 3, which is proved in the Appendix. Therefore, we derive

$$
-(-1)^{\sum_{i=1}^{d} \int \boldsymbol{\Delta}_{n-1}^{i-1} \cup_{n-2} \Delta_{n-1}^{i}}=(-1)^{\int_{w_{2}} \Delta_{n-2}} .
$$

Lemma 3. In $n$-dimension manifold with triangulation and branching structure, the homology class of $w_{2}$ can be represented by a $(n-2)$-chain $w_{2} \in C_{n-2}\left(M_{n}, \mathbb{Z}_{2}\right)$,

$$
w_{2}=\sum_{\Delta_{n-2}} c\left(\Delta_{n-2}\right) \Delta_{n-2},
$$

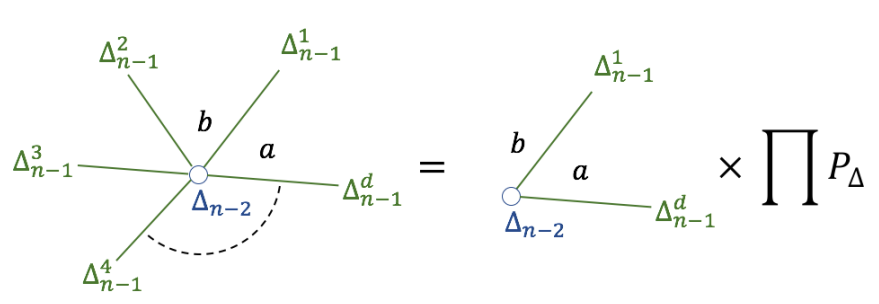

FIG. 4. By the operations defined in Fig. 3, we can simplify the product $S_{\Delta_{n-1}^{d}} \cdots S_{\Delta_{n-1}^{2}} S_{\Delta_{n-1}^{1}}=S_{\Delta_{n-1}^{d}} S_{\Delta_{n-1}^{1}} \prod_{\Delta_{n} \neq a, b} \times$ $P_{\Delta_{n}}^{\int \boldsymbol{\Delta}_{n-2} \cup_{n-2} \boldsymbol{\Delta}_{n}+\boldsymbol{\Delta}_{n} \cup_{n-2} \boldsymbol{\Delta}_{n-2}}$. 
where

$$
\begin{aligned}
c\left(\Delta_{n-2}\right)= & 1+\sum_{- \text {oriented } \Delta_{n}=\langle 0 \ldots n\rangle} \sum_{j_{1}<j_{2} \mid j_{1}, j_{2} \in \text { even }} \\
& \times \Delta_{n-2}\left(\left\langle 0 \cdots \hat{j_{1}} \cdots \hat{j_{2}} \cdots n\right\rangle\right) \\
& +\sum_{+ \text {oriented } \Delta_{n}=\langle 0 \ldots n\rangle} \sum_{k_{1}<k_{2} \mid k_{1}, k_{2} \in \text { odd }} \\
& \times \Delta_{n-2}\left(\left\langle 0 \cdots \hat{k_{1}} \cdots \hat{k}_{2} \cdots n\right\rangle\right) .
\end{aligned}
$$

We can modify the sign of $S_{\Delta_{n-1}}$ as

$$
S_{\Delta_{n-1}}^{E} \equiv(-1)^{\int_{E} \Delta_{n-1}} S_{\Delta_{n-1}},
$$

where $E \in C_{n-1}\left(M_{n}, \mathbb{Z}_{2}\right)$ is a choice of spin structure satisfying $\partial E=w_{2}$. In these modified operators, the constraint on the fermionic operator becomes

$$
S_{\delta \Delta_{n-2}}^{E} \prod_{\Delta_{n}} P_{\Delta_{n}}^{\int \boldsymbol{\Delta}_{n-2} \cup_{n-2} \boldsymbol{\Delta}_{n}+\boldsymbol{\Delta}_{n} \cup_{n-2} \boldsymbol{\Delta}_{n-2}}=1,
$$

which is mapped to

$$
\begin{aligned}
G_{\Delta_{n-2}} & =U_{\delta \Delta_{n-2}} \prod_{\Delta_{n}} W_{\Delta_{n}}^{\int \boldsymbol{\Delta}_{n-2} \cup_{n-2} \boldsymbol{\Delta}_{n}+\boldsymbol{\Delta}_{n} \cup_{n-2} \boldsymbol{\Delta}_{n-2}} \\
& =\prod_{\Delta_{n-1} \supset \Delta_{n-2}} X_{\Delta_{n-1}}\left(\prod_{\Delta_{n-1}{ }^{\prime}} Z_{\Delta_{n-1}{ }^{\prime}}^{\int \delta \boldsymbol{\Delta}_{n-2} \cup_{n-2} \boldsymbol{\Delta}_{n-1}{ }^{\prime}}\right),
\end{aligned}
$$

where $U_{\lambda_{n-1}}$ is defined by

$$
U_{\lambda_{n-1}+\lambda_{n-1}^{\prime}} \equiv(-1)^{\int \lambda_{n-1} \cup_{n-2} \lambda_{n-1}^{\prime}} U_{\lambda_{n-1}^{\prime}} U_{\lambda_{n-1}},
$$

and it can be calculated directly from $U_{\Delta_{n-1}}$ defined in Eq. (23):

$$
U_{\lambda_{n-1}} \equiv \prod_{\Delta_{n-1} \mid \lambda\left(\Delta_{n-1}\right)=1} X_{\Delta_{n-1}}\left(\prod_{\Delta_{n-1}{ }^{\prime}} Z_{\Delta_{n-1}}^{\int \Delta_{n-1}{ }^{\prime} \cup_{n-2} \lambda_{n-1}}\right) .
$$

We need to impose this gauge constraint $G_{\Delta_{n-2}}=1$ on bosonic operators for every $(n-2)$-simplex $\Delta_{n-2}$.

We also need to impose the even total parity constraint for fermions,

$$
\prod_{\Delta_{n}} P_{\Delta_{n}}=1,
$$

since it is mapped to the bosonic operator $\prod_{\Delta_{n}} W_{\Delta_{n}}=1$. After imposing the gauge constraints, the $n$-dimensional bosonfermion duality Eq. (23) is completed.

\section{MODIFIED GAUSS'S LAW AND EUCLIDEAN ACTION}

\section{A. Gauss's law as boundary anomaly}

First, we consider the standard $(n-1)$-form $\mathbb{Z}_{2}$ lattice gauge theory on the $n$-dimensional manifold $M_{n}$,

$$
H^{0}=-J_{1} \sum_{\Delta_{n-1}} X_{\Delta_{n-1}}-J_{2} \sum_{\Delta_{n}} W_{\Delta_{n}},
$$

with the gauge constraint (Gauss's law):

$$
G_{\Delta_{n-2}}^{0}=\prod_{\Delta_{n-1} \supset \Delta_{n-2}} X_{\Delta_{n-2}}=1 \text {. }
$$

It is well known that its Euclidean theory is $(n+1)$ dimensional Ising model (with a certain choice of $J_{1}$ and $J_{2}$ ) [14],

$$
S_{\text {Ising }}\left(A_{n-1}\right)=-J \sum_{\Delta_{n} \subset Y}\left|\delta A_{n-1}\left(\Delta_{n}\right)\right|,
$$

where $A \in C^{n-1}\left(Y, \mathbb{Z}_{2}\right)$ is a $(n-1)$-cochain on the spacetime manifold $Y,|\delta A|=0,1$ gives $\delta A(\bmod 2)$, and $J$ depends on $J_{1}$ and $J_{2}$. In this case, $S_{\text {Ising }}$ is invariant under the gauge transformation $A_{n-1} \rightarrow A_{n-1}+\delta \Lambda_{n-2}$ for arbitrary $(n-2)$-cochain $\Lambda_{n-2} \in C^{n-2}\left(Y, \mathbb{Z}_{2}\right)$. Therefore, $S_{\text {Ising }}$ has no boundary anomaly under the standard Gauss's law.

Now, we propose a class of $\mathbb{Z}_{2}$ lattice gauge theory,

$$
H=-J_{1} \sum_{\Delta_{n-1}} U_{\Delta_{n-1}}-J_{2} \sum_{\Delta_{n}} W_{\Delta_{n}},
$$

with the modified Gauss's law (gauge constraints) at ( $n-2)$ simplices:

$$
G_{\Delta_{n-2}}=\prod_{\Delta_{n-1} \supset \Delta_{n-2}} X_{\Delta_{n-1}}\left(\prod_{\Delta_{n-1}{ }^{\prime}} Z_{\Delta_{n-1}}^{\int \delta \boldsymbol{\Delta}_{n-2} \cup_{n-2} \Delta_{n-1}}\right)=1 .
$$

This model describes a free fermion system, since it is dual to

$$
\begin{aligned}
H_{f} & =-J_{1} \sum_{\Delta_{n-1}}(-1)^{\int_{E} \Delta_{n-1}} i \gamma_{L\left(\Delta_{n-1}\right)} \gamma_{R\left(\Delta_{n-1}\right)}^{\prime}-J_{2} \sum_{\Delta_{n}}\left(-i \gamma_{\Delta_{n}} \gamma_{\Delta_{n}}^{\prime}\right) \\
& =-J_{1} \sum_{\Delta_{n-1}} S_{\Delta_{n-1}}^{E}-J_{2} \sum_{\Delta_{n}} P_{\Delta_{n}} .
\end{aligned}
$$

The modified Gauss's law Eq. (52) on a $(n-2)$-simplex $\Delta_{n-2}$, or equivalently on the dual $(n-2)$-cochain $\boldsymbol{\Delta}_{n-2}$, can be generalized to an arbitrary $(n-2)$-cochain $\lambda_{n-2}=$ $\sum_{i} \boldsymbol{\Delta}_{n-2}^{i}$; the Gauss's law is

$$
\begin{aligned}
1= & G_{\lambda_{n-2}}=\prod_{i} G_{\Delta_{n-2}^{i}} \\
= & \left(\prod_{\Delta_{n-1} \in \delta \lambda_{n-2}} X_{\Delta_{n-1}}\right)\left(\prod_{\Delta_{n-1}{ }^{\prime}} Z_{\Delta_{n-1}{ }^{\prime} \delta \lambda_{n-2} \cup_{n-2} \Delta_{n-1}{ }^{\prime}}\right) \\
& \times(-1)^{\int \lambda_{n-2} \cup_{n-4} \lambda_{n-2}+\lambda_{n-2} \cup_{n-3} \delta \lambda_{n-2},}
\end{aligned}
$$

where the sign comes from anticommutation of $X$ and $Z$ on the same simplex. This can be proved by induction.

(1) We first check for $\lambda_{n-2}=\boldsymbol{\Delta}_{n-2}$, where $\lambda_{n-2}$ contains a single $(n-2)$-simplex. We have $\boldsymbol{\Delta}_{n-2} \cup_{n-4} \boldsymbol{\Delta}_{n-2}+$ $\boldsymbol{\Delta}_{n-2} \cup_{n-3} \delta \boldsymbol{\Delta}_{n-2}=0$ by the definition of higher cup products since the vertices in (8) cannot match. For example, $\boldsymbol{\Delta}_{n-2}$ acts only nontrivial on a $(n-2)$-simplex with $(n-1)$ vertices, while $\boldsymbol{\Delta}_{n-2} \cup_{n-4} \boldsymbol{\Delta}_{n-2}$ has the input of $(n+1)$ vertices, which has 2 extra vertices at least. $\boldsymbol{\Delta}_{n-2}$ vanishes when it acts on any simplex with the extra vertices. The gauge constraint reduces the original form Eq. (52).

(2) It is straightforward to check $G_{\lambda_{n-2}} G_{\lambda_{n-2}^{\prime}}=G_{\lambda_{n-2}+\lambda_{n-2}^{\prime}}$ using the recursive property of cup products:

$$
\alpha \cup_{a} \beta+\beta \cup_{a} \alpha=\alpha \cup_{a+1} \delta \beta+\delta \alpha \cup_{a+1} \beta+\delta\left(\alpha \cup_{a+1} \beta\right) .
$$


Consider now the following $(n-1)$-form gauge theory defined on a general triangulated $(n+1)$-dimensional manifold $Y$ :

$$
\begin{aligned}
S\left(A_{n-1}\right)= & -\sum_{\Delta_{n} \subset Y}\left|\delta A_{n-1}\left(\Delta_{n}\right)\right|+i \pi \int_{Y}\left(A_{n-1} \cup_{n-3} A_{n-1}\right. \\
& \left.+A_{n-1} \cup_{n-2} \delta A_{n-1}\right)
\end{aligned}
$$

where $A_{n-1} \in C^{n-1}\left(Y, \mathbb{Z}_{2}\right)$ and the gauge symmetry acts by $A_{n-1} \rightarrow A_{n-1}+\delta \Lambda_{n-2}$ for $\Lambda_{n-2} \in C^{n-2}\left(Y, \mathbb{Z}_{2}\right)$. The second term is the generalized Steenrod square defined in Ref. [5]. The action is gauge invariant up to a boundary term

$$
\begin{aligned}
& S\left(A_{n-1}+\delta \Lambda_{n-2}\right)-S\left(A_{n-1}\right) \\
& =i \pi \int_{\partial Y}\left(\Lambda_{n-2} \cup_{n-4} \Lambda_{n-2}+\Lambda_{n-2} \cup_{n-3} \delta \Lambda_{n-2}\right. \\
& \left.\quad+\delta \Lambda_{n-2} \cup_{n-2} A_{n-1}\right) \\
& =i \pi \int_{\partial Y}\left(\Lambda \cup_{n-4} \Lambda+\Lambda \cup_{n-3} \delta \Lambda+\delta \Lambda \cup_{n-2} A\right),
\end{aligned}
$$

where we have omitted the subscript of $A_{n-1}$ and $\Lambda_{n-2}$ for simplicity. This boundary term determines the Gauss's law for the wave-function $\Psi(A)$ on the spatial slice $M=\partial Y$,

$$
\Psi(A+\delta \Lambda)=(-1)^{\omega(\Lambda, A)} \Psi(A),
$$

where $\quad \omega(\Lambda, A)=\int_{M}\left(\Lambda \cup_{n-4} \Lambda+\Lambda \cup_{n-3} \delta \Lambda+\delta \Lambda \cup_{n-2}\right.$ $A)$. The Gauss's law is the same as the gauge constraint Eq. (54) if we identify $Z_{\Delta_{n-1}}$ as $(-1)^{A_{n-1}\left(\Delta_{n-1}\right)}$ and $X_{\Delta_{n-1}}$ acts as the transformation $A_{n-1} \rightarrow A_{n-1}+\boldsymbol{\Delta}_{n-1}$. The modified Gauss's law Eq. (52) represents the boundary anomaly of topological action Eq. (56) as we claimed.

In the following subsection, we derive the Euclidean action of the modified $\mathbb{Z}_{2}$ lattice gauge theory Eq. (51) explicitly, which is analogous to Eq. (56).

\section{B. Euclidean path integral of lattice gauge theories}

Start with the Hamiltonian of modified $\mathbb{Z}_{2}$ lattice gauge theory,

$$
\begin{aligned}
H= & -J_{1} \sum_{\Delta_{n-1}} U_{\Delta_{n-1}}-J_{2} \sum_{\Delta_{n}} W_{\Delta_{n}} \\
= & -J_{1} \sum_{\Delta_{n-1}} X_{\Delta_{n-1}}\left(\prod_{\Delta_{n-1}{ }^{\prime}} Z_{\Delta_{n-1}}^{\int \Delta_{n-1}{ }^{\prime} \cup_{n-2} \Delta_{n-1}}\right) \\
& -J_{2} \sum_{\Delta_{n}} \prod_{\Delta_{n-1} \subset \Delta_{n}} Z_{\Delta_{n-1}},
\end{aligned}
$$

with gauge constraints

$$
G_{\Delta_{n-2}}=\prod_{\Delta_{n-1} \supset \Delta_{n-2}} X_{\Delta_{n-1}}\left(\prod_{\Delta_{n-1}{ }^{\prime}} Z_{\Delta_{n-1}{ }^{\prime}}^{\int \delta \Delta_{n-2} \cup_{n-2} \Delta_{n-1}{ }^{\prime}}\right)=1 .
$$

The partition function is

$$
\mathcal{Z}=\operatorname{Tr} e^{-\beta H}=\operatorname{Tr} T^{M},
$$

where we use Trotter-Suzuki decomposition in the imaginary time direction and $T$ is the transfer matrix defined as

$$
T=\left(\prod_{\Delta_{n-2}} \delta_{G_{\Delta_{n-2}}, 1}\right) e^{-\delta \tau H}
$$

The first factor arises from the gauge constraints on the Hilbert space. The space-time manifold consists of many time slices labeled by layers $\{i\}$. In the $i$ th layer, we insert a complete basis (in Pauli matrix $\left.Z_{\Delta_{n-1}}\right): b_{n-1}^{i} \in C^{n-1}\left(M_{n}, \mathbb{Z}_{2}\right)\left(\mathrm{a} \mathbb{Z}_{2}\right.$ field on each $\Delta_{n-1}$ of the spatial manifold $M_{n}$ such that $Z_{\Delta_{n-1}}=$ $\left.(-1)^{b_{n-1}^{i}\left(\Delta_{n-1}\right)}\right)$. The transfer matrix $T$ between the $i$ th layer and the $(i+1)$ th layer contains gauge constraints on every spatial $(n-2)$-simplex $\Delta_{n-2}$ :

$$
\delta_{G_{\Delta_{n-2}}, 1}=\frac{1+G_{\Delta_{n-2}}}{2}=\frac{1}{2} \sum_{a_{n-2}^{i+1 / 2}=0,1}\left(G_{\Delta_{n-2}}\right)^{a_{n-2}^{i+1 / 2}},
$$

where we introduce the Lagrangian multiplier $a_{n-2}^{i+1 / 2} \in$ $C^{n-2}\left(M_{n}, \mathbb{Z}_{2}\right)$ (a $\mathbb{Z}_{2}$ field living on each $\Delta_{n-2}$ of the spatial manifold $M_{n}$ ). Notice that $a_{n-2}^{i+1 / 2}$ defined between two time slices lives on the spatial $(n-2)$-simplex $\Delta_{n-2}$, which can be interpreted as the space-time $(n-1)$-simplex between the two layers. From the similar calculation in Ref. [2], we have

$$
\mathcal{Z}=\sum_{\left\{a_{n-2}^{i+1 / 2, b_{n-1}^{i}}\right\}} \exp \left(\left[S_{\text {Ising }}+S_{\text {top }}\right]\left\{a_{n-2}^{i+1 / 2}, b_{n-1}^{i}\right\}\right)
$$

where

$$
S_{\text {Ising }}\left\{a_{n-2}^{i+1 / 2}, b_{n-1}^{i}\right\}=\sum_{i}\left(-J_{s} \sum_{\Delta_{n}}\left|\delta b_{n-1}^{i}\left(\Delta_{n}\right)\right|-J_{\tau} \sum_{\Delta_{n-1}}\left|\left[b_{n-1}^{i}+b_{n-1}^{i+1}+\delta a_{n-2}^{i+1 / 2}\right]\left(\Delta_{n-1}\right)\right|\right)
$$

and

$$
\begin{aligned}
S_{\text {top }}\left\{a_{n-2}^{i+1 / 2}, b_{n-1}^{i}\right\}= & i \pi \sum_{i} \int_{M_{n}} a_{n-2}^{i+1 / 2} \cup_{n-4} a_{n-2}^{i+1 / 2}+a_{n-2}^{i+1 / 2} \cup_{n-3} \delta a_{n-2}^{i+1 / 2}+\delta a_{n-2}^{i+1 / 2} \cup_{n-2} b_{n-1}^{i+1} \\
& +b_{n-1}^{i} \cup_{n-2}\left(b_{n-1}^{i}+b_{n-1}^{i+1}+\delta a_{n-2}^{i+1 / 2}\right) .
\end{aligned}
$$


Here $J_{s}, J_{\tau}$ are constants depending on $J_{1}, J_{2}, \delta \tau$ in the original Hamiltonian and we assume $J_{s}=J_{\tau}=J$ for simplicity. $|\cdots|$ gives the argument's parity 0 or 1 . The gauge transformations act as

$$
\begin{aligned}
b_{n-1}^{i} & \rightarrow b_{n-1}^{i}+\delta \lambda^{i}, \\
a_{n-2}^{i+1 / 2} & \rightarrow a_{n-2}^{i+1 / 2}+\delta \mu^{i}+\lambda^{i}+\lambda^{i+1},
\end{aligned}
$$

where $\lambda^{i}$ are arbitrary $(n-2)$-cochains and $\mu^{i}$ are arbitrary $(n-3)$-cochains.

If we interpret $a_{n-2}^{i+1 / 2}$ as space-time $(n-1)$-cochains, we can rewrite

$$
\left\{a_{n-2}^{i+1 / 2}, b_{n-1}^{i}\right\} \rightarrow A_{n-1} \in C^{n-1}\left(Y, \mathbb{Z}_{2}\right)
$$

which is a $\mathbb{Z}_{2}$ field living on $(n-1)$-simplices in space-time manifold $Y$. It is natural to write $S_{\text {Ising }}$ in Eq. (65) as

$$
S_{\text {Ising }}=-\sum_{\Delta_{n} \subset Y}\left|\delta A_{n-1}\left(\Delta_{n}\right)\right| .
$$

The space-time manifold $Y=M_{n} \times[-\infty, 0]$ (spatial and temporal parts) is not a triangulation, since we only triangularize the spatial manifold $M_{n}$ under the discretized time. The (higher) cup products are not well-defined in $Y$. However, we can still write an expression

$$
S_{\text {top }}=i \pi \int_{Y^{\prime}}\left(A_{n-1} \cup_{n-3} A_{n-1}+A_{n-1} \cup_{n-2} \delta A_{n-1}\right)
$$

in $(n+1)$-dimensional triangulation $Y^{\prime}$ such that $Y^{\prime}$ is a refinement of $Y$. We can check that Eqs. (66) and (70) produce the same boundary term under gauge transformations.

\section{CONCLUSIONS}

We have extended the the exact bosonization Eq. (21) in 2D and Eq. (22) in 3D to arbitrary dimensions. The dictionary for $n$-dimensional boson-fermion duality is given in Eq. (23). This bosonization is a duality between any fermionic system in arbitrary $n$ spatial dimensions and $(n-1)$-form $\mathbb{Z}_{2}$ gauge theories in $n$ dimensions with gauge constraints (the modified Gauss's law). This map preserves locality: Every local even fermionic observable is mapped to a local gauge-invariant bosonic operator. The formula has an explicit dependence on the second Stiefel-Whitney class of the manifold and a choice of spin structure is needed. As a side product, we discover formula Eq. (29) for Stiefel-Whitney homology classes on lattices. In the Euclidean picture, we have shown that the Euclidean path integral of the $n$-dimensional $\mathbb{Z}_{2}$ gauge theory with modified Gauss's law is the $(n+1)$-dimensional Ising model with an additional topological Steenrod square Eq. (56) term.

\section{ACKNOWLEDGMENTS}

Y.C. thanks Po-Shen Hsin and his advisor Prof. Anton Kapustin for many very helpful discussions. Y.C. also thanks Tyler Ellison and Nathanan Tantivasadakarn for their useful feedback. This research was supported in part by the US Department of Energy, Office of Science, Office of High Energy Physics, under Award No. de-sc0011632. Anton Kapustin was also supported by the Simons Investigator Award.

\section{APPENDIX: A FORMULA FOR STIEFEL-WHITNEY HOMOLOGY CLASSES}

In this section, we prove Lemma 3, Eq. (41). First, let us recall the theorem proved in Ref. [15]. Let $s$ be a $p$-simplex, say $s=\left\langle v_{0}, v_{1}, \ldots, v_{p}\right\rangle$. Let $k$ be another simplex which has $\mathrm{s}$ as a face; i.e., $s \subset k$ ( $s$ may be equal to $k)$. Let

$$
\begin{aligned}
B_{-1} & =\text { set of vertices of } k \text { less than } v_{0}, \\
B_{0} & =\text { set of vertices of } k \text { between } v_{0} \text { and } v_{1}, \\
B_{m} & =\text { set of vertices of } k \text { between } v_{m} \text { and } v_{m+1}, \\
B_{p} & =\text { set of vertices of } k \text { greater than } v_{p} .
\end{aligned}
$$

We say that $s$ is regular in $k$, if $\#\left(B_{m}\right)=0$ for every odd $m$. Let $\partial_{p}(k)$ denote the $\bmod 2$ chain which consists of all $p$-dimensional simplices $s$ in $k$ so $s$ is regular in $k$. For example, $\langle 012\rangle$ and $\langle 023\rangle$ are regular in $\langle 0123\rangle$ and therefore $\partial_{2}(\langle 0123\rangle)=\langle 012\rangle+\langle 023\rangle$. The theorem is [15]

Theorem 1. $\sum_{k \mid \operatorname{dim} k \geqslant(n-2)} \partial_{n-2}(k)$ is a $(n-2)$-chain which represents $w_{2}$.

In particular, for any $n^{\prime}$-simplex $\Delta_{n^{\prime}}=\left\langle 0 \ldots n^{\prime}\right\rangle$, all $\left(n^{\prime}-1\right)$-simplices regular in $\Delta_{n^{\prime}}$ are

$$
\langle 0 \ldots \hat{i} \ldots n\rangle \forall i \in \text { odd }
$$

and all $\left(n^{\prime}-2\right)$-simplices regular in $\Delta_{n^{\prime}}$ are

$$
\langle 0 \ldots \hat{i} \ldots \hat{j} \ldots n\rangle \forall i \in \text { odd, } j \in \text { even, } i<j .
$$

We now use this theorem to prove Lemma 3.

Proof of Lemma 3. For every $(n-2)$-simplex $\Delta_{n-2}$, it is regular in itself. This contributes the 1 in the coefficient of $c\left(\Delta_{n-2}\right)$ in Eq. (41).

For every $(n-1)$-simplex $\Delta_{n-1}$, it is a boundary of two $n$-simplices $\Delta_{n}^{L}$ and $\Delta_{n}^{R}$, with $\Delta_{n-1}$ being an outward boundary of $\Delta_{n}^{L}$ and an inward boundary of $\Delta_{n}^{R}$. We define that $\Delta_{n-1}$ belongs to $\Delta_{n}^{R}$ and the summation of $\operatorname{dim} k=n-1, n$ in Theorem 1 can be written as

$$
\begin{aligned}
& \sum_{\Delta_{n-1}} \partial_{n-2}\left(\Delta_{n-1}\right)+\sum_{\Delta_{n}} \partial_{n-2}\left(\Delta_{n}\right) \\
& =\sum_{\Delta_{n}}\left[\partial_{n-2}\left(\Delta_{n}\right)+\sum_{\Delta_{n-1} \in \Delta_{n} \mid \Delta_{n-1} \text { is inward }} \partial_{n-2}\left(\Delta_{n-1}\right)\right] .
\end{aligned}
$$

If $\Delta_{n}=\langle 0 \ldots n\rangle$, is + oriented, the terms in the summation are

$$
\begin{aligned}
& \partial_{n-2}(\langle 0 \ldots n\rangle)+\sum_{0 \leqslant i \leqslant n \mid i \in \text { odd }} \partial_{n-2}(\langle 0 \ldots \hat{i} \ldots n\rangle) \\
& =\sum_{i, j \mid i<j, i \in \text { odd }, j \in \text { even }}\langle 0 \ldots \hat{i} \ldots \hat{j} \ldots n\rangle \\
& +\sum_{0 \leqslant i \leqslant n \mid i \in \text { odd }}\left(\sum_{j<i \mid j \in \text { odd }}\langle 0 \ldots \hat{j} \ldots \hat{i} \ldots n\rangle\right. \\
& \left.+\sum_{j>i \mid j \in \text { even }}\langle 0 \ldots \hat{i} \ldots \hat{j} \ldots n\rangle\right) \\
& =\sum_{i, j \mid i<j, i \in \text { odd }, j \in \text { odd }}\langle 0 \ldots \hat{i} \ldots \hat{j} \ldots n\rangle,
\end{aligned}
$$


where we have used the definition of regular simplex defined above. Similarly, we can derive that if $\Delta_{n}=\langle 0 \ldots n\rangle$ is - oriented, the term is

$$
\sum_{i, j \mid i<j, i \in \text { even }, j \in \text { even }}\langle 0 \ldots \hat{i} \ldots \hat{j} \ldots n\rangle .
$$

Combining Eqs. (A5) and (A6) with the 1 from $\operatorname{dim} k=n-2$ in Theorem 1, we have

$$
w_{2}=\sum_{\Delta_{n-2}} c\left(\Delta_{n-2}\right) \Delta_{n-2},
$$

where

$$
\begin{aligned}
c\left(\Delta_{n-2}\right)= & +\sum_{- \text {oriented } \Delta_{n}=\langle 0 \ldots n\rangle} \sum_{j_{1}<j_{2} \mid j_{1}, j_{2} \in \text { even }} \Delta_{n-2}\left(\left\langle 0 \cdots \hat{j_{1}} \cdots \hat{j_{2}} \cdots n\right\rangle\right) \\
& +\sum_{+ \text {oriented } \Delta_{n}=\langle 0 \ldots n\rangle k_{1}<k_{2} \mid k_{1}, k_{2} \in \text { odd }} \boldsymbol{\Delta}_{n-2}\left(\left\langle 0 \cdots \hat{k_{1}} \cdots \hat{k_{2}} \cdots n\right\rangle\right) .
\end{aligned}
$$

[1] Y.-A. Chen, A. Kapustin, and D. Radičević, Exact bosonization in two spatial dimensions and a new class of lattice gauge theories, Ann. Phys. 393, 234 (2018).

[2] Y.-A. Chen and A. Kapustin, Bosonization in three spatial dimensions and a 2-form gauge theory, Phys. Rev. B 100, 245127 (2019).

[3] D. Gaiotto and A. Kapustin, Spin TQFTs and fermionic phases of matter, Int. J. Mod. Phys. A 31, 1645044 (2016).

[4] N. E. Steenrod, Products of cocycles and extensions of mappings, Ann. Math. 48, 290 (1947).

[5] T. Lan, C. Zhu, and X.-G. Wen, Fermion decoration construction of symmetry protected trivial orders for fermion systems with any symmetries $G_{f}$ and in any dimensions, Phys. Rev. B 100, 235141 (2019).

[6] A. Kapustin and R. Thorngren, Fermionic SPT phases in higher dimensions and bosonization, J. High Energy Phys. 10 (2017) 080 .

[7] X.-G. Wen, Exactly soluble local bosonic cocycle models, statistical transmutation, and simplest time-reversal symmetric topological orders in 3+1 dimensions, Phys. Rev. B 95, 205142 (2017).

[8] Y.-A. Chen, T. D. Ellison, and N. Tantivasadakarn, Disentangling supercohomology symmetry-protected topological phases in three spatial dimensions, arXiv:2008.05652.

[9] S. B. Bravyi and A. Yu. Kitaev, Fermionic quantum computation, Ann. Phys. 298, 210 (2002).

[10] R. C. Ball, Fermions without Fermion Fields, Phys. Rev. Lett. 95, 176407 (2005).

[11] F. Verstraete and J. I. Cirac, Mapping local Hamiltonians of fermions to local Hamiltonians of spins, J. Stat. Mech. (2005) P09012.

[12] T. Banks, Fermi/Pauli duality in arbitrary dimension, arXiv:1908.10453.

[13] S. Tata, Geometrically interpreting higher cup products, and application to combinatorial pin structures, arXiv:2008.10170.

[14] J. B. Kogut, An introduction to lattice gauge theory and spin systems, Rev. Mod. Phys. 51, 659 (1979).

[15] R. Z. Goldstein and E. C. Turner, A formula for Stiefel-Whitney homology classes, Proc. Am. Math. Soc. 58, 339 (1976). 